\title{
An SiC/GaN Detector/Front-End Detection System for High-Resolution Alpha-Particle Spectroscopy
}

\author{
A. Pullia, G. Bertuccio, D. Maiocchi, S. Caccia, and F. Zocca
}

\begin{abstract}
An $\alpha$-particle spectrometer has been assembled, consisting of an epitaxial $50 \mu$ m thick $4 \mathrm{H}$ silicon carbide $(\mathrm{SiC})$ detector connected to a gallium-nitride $(\mathrm{GaN})$ high-electron mobility transistor (HEMT) used as input transistor of the front-end electronics. The depleted layer of the $\mathrm{SiC}$ diode detector was sufficient to stop all $\alpha$ particles in the $4.8-\mathrm{MeV}$ to $5.8 \mathrm{-MeV}$ energy range. An excellent energy resolution of $\sim 0.9 \%$ has been obtained in this energy range at a temperature of $55^{\circ} \mathrm{C}$. The energy-resolution limiting factor is found to be the dispersion of the energy loss in the gold Schottky contact, which acts as entrance window to the detector. We used a GaN front-end transistor because this material offers two important advantages over silicon: 1) it is a wide bandgap semiconductor and therefore is intrinsically more desirable for room and above-room temperature operation and 2) it can be grown on $\mathrm{SiC}$ substrates so as to realize $\mathrm{SiC} / \mathrm{GaN}$ integrated systems. SiC-detector spectrometers are interesting in many nuclear applications where the operation environment is hostile, both in terms of ionising radiations and of high temperatures. Such applications include monitoring of ionising radiations in nuclear power plants and beam diagnostic in fundamental nuclear physics experiments.
\end{abstract}

Index Terms-Alpha spectroscopy, gallium nitride (GaN), silicon carbide (SiC).

\section{INTRODUCTION}

$\mathbf{R}$ ECENT technological advances permit fabrication of $>50-\mu \mathrm{m}$-thick epitaxial silicon carbide layers with a low doping concentration of $<10^{14} \mathrm{~cm}^{-3}$ and a low defect concentration [1]. It has been demonstrated that Schottky diodes realized on these layers work well as detectors of ionizing particles and radiations [2]-[4]. The bandgap energy and the mean pair-production energy for free carriers of $\mathrm{SiC}$ are shown in Table I along with those of some other semiconductors. Thanks to the relatively wide bandgap of silicon carbide, the leakage current of $\mathrm{SiC}$ diodes is exceptionally low, of the order of $5 \mathrm{pA} / \mathrm{cm}^{2}$ at room temperature with a bias voltage as high as $100 \mathrm{kV} / \mathrm{cm}$ [5]. Therefore, $\mathrm{SiC}$ detectors feature an ultra-low intrinsic noise and a fast response. In Fig. 1, it can be seen that the leakage current of $\mathrm{SiC}$ detectors is almost three orders of

Manuscript received July 02, 2008; revised September 25, 2008. Current version published December 31, 2008. This work was supported by the Italian Ministry of University and Scientific Research under PRIN 2004 Project "Development of a SiC/GaN Detector/Front-end Integrated System for Ionizing Radiation Spectroscopy at Room and High Temperatures

A. Pullia and F. Zocca are with the University of Milan, Department of Physics and Istituto Nazionale di Fisica Nucleare, Milan 20133, Italy.

G. Bertuccio and S. Caccia are with the Politechnic of Milan and also with Istituto Nazionale di Fisica Nucleare, Milan 20133, Italy.

D. Maiocchi was with the University of Milan, Department of Physics, Milan 20133, Italy. He is now with MAXIM Corp , Rozzano 20089, Italy.

Digital Object Identifier 10.1109/TNS.2008.2007722

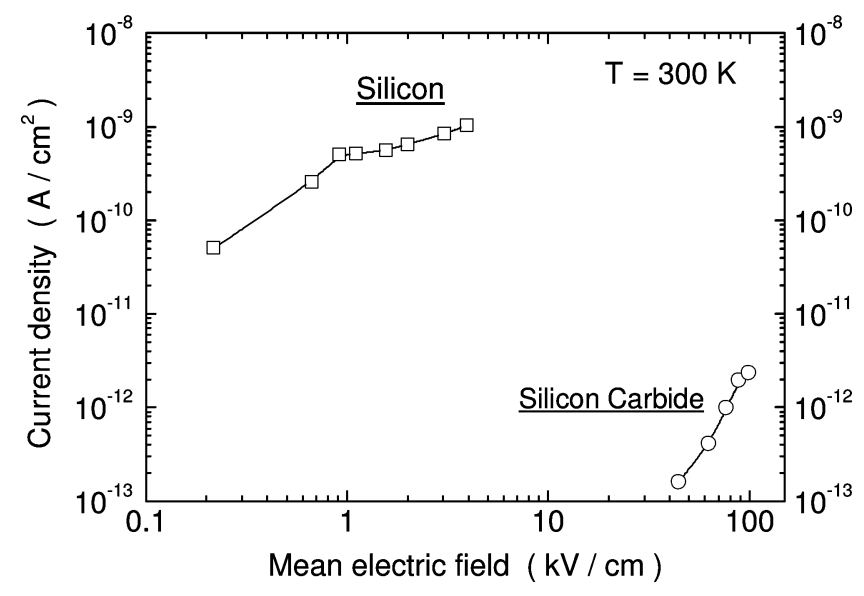

Fig. 1. Density of the typical leakage current of $\mathrm{SiC}$ and $\mathrm{Si}$ diodes under a reverse bias voltage. The $\mathrm{SiC}$ detectors show much lower leakage current at much higher electric fields.

magnitude lower than that of Si detectors, even when biased at much higher electric fields. This property makes $\mathrm{SiC}$ detectors particularly suited for room or above-room temperature operation, where the shot noise of the detector leakage current is normally the limiting factor of the detector performance. It has been shown that by using state-of-the-art front-end electronics based on silicon devices, the performance of $\mathrm{SiC}$ detectors at high temperatures is limited by the electronics itself rather than the detector [5]. In this paper, we studied the possibility of using front-end electronics realized in gallium nitride, which has a wider bandgap than silicon (i.e., $3.2 \mathrm{eV}$ ) and should therefore be more useful for above-room-temperature operation. GaN electronic devices can moreover be integrated on $\mathrm{SiC}$ substrates, which opens the way to fully integrated $\mathrm{SiC} / \mathrm{GaN}$ detector/electronic systems [6]. The integration of GaN circuits is currently an emerging technology mostly used for radio-frequency circuit applications, such as radars and broadband telecommunications.

\section{HyBRID SIC/GAN SYSTEM}

In Fig. 2, the top view and a cross section of the available detectors are shown. Each detector consists of an $n+$ conductive $\mathrm{SiC}$ bulk with a $50-\mu \mathrm{m}$ epitaxial layer grown upon. A circular gold Schottky contact is realized on the top surface, surrounded by two guard rings, and a common Ti-Pt-Au ohmic contact is realized on the bottom surface. The Au rectifying contact has a thickness of $1000 \AA$ and acts as entrance window to the detector for the impinging $\alpha$ rays. Four detectors were available with active areas in the $0.63-\mathrm{mm}^{2}$ to $1.6-\mathrm{mm}^{2}$ range. Fig. 3 shows the leakage current of these detectors as being measured at room 
TABLE I

SEMICONDUCTOR PHYSICAL PROPERTIES

\begin{tabular}{cccc}
\hline Semiconductor & $\begin{array}{c}\text { Band-gap } \\
\text { energy (eV) }\end{array}$ & $\begin{array}{c}\text { Pair-production } \\
\text { energy }(\mathrm{eV})\end{array}$ & $\begin{array}{c}\text { Relative } \\
\text { permittivity } \varepsilon_{\mathrm{r}}\end{array}$ \\
\hline 4H SiC & 3.25 & $7.7^{*}$ & 10.0 \\
\hline Diamond & 5.5 & 13.1 & 5.7 \\
\hline $\mathrm{Si}$ & 1.12 & 3.6 & 11.7 \\
\hline Ge & 0.67 & 2.9 & 16.0 \\
\hline $\begin{array}{l}\text { * Ref. [3] } \\
\text { ** Requires cryogeny }\end{array}$ & & &
\end{tabular}

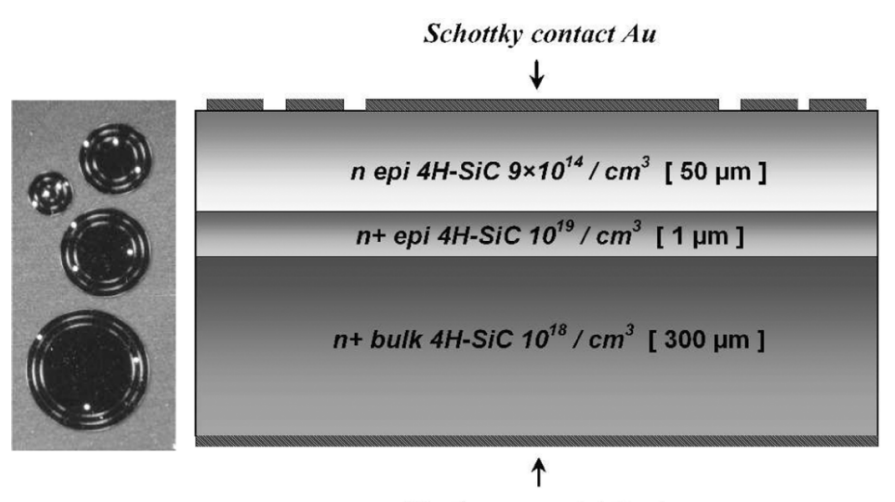

Ohmic contact Ti-Pt-Au

Fig. 2. Front view and cross-section of the $\mathrm{SiC}$ diodes used in this work as alpha-particle detectors. The doping concentration (nitrogen) in the $\mathrm{SiC}$ layers is shown. The Schottky contact is realized with a $1000 \AA$ Au contact.

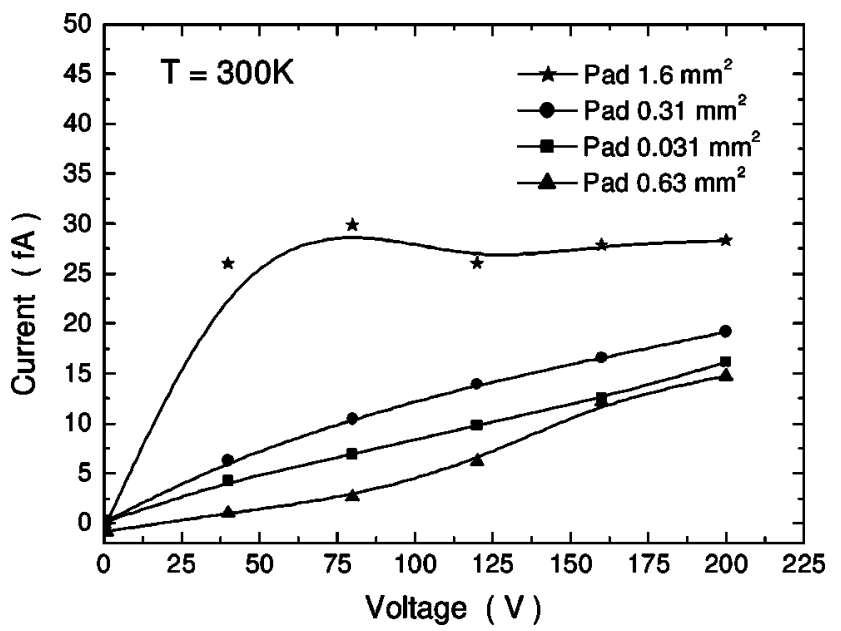

Fig. 3. Measured leakage current of the four reverse-biased diode detectors of Fig. 2. The current is exceptionally low.

temperature with a sophisticated instrument. It can be seen that the extremely low leakage current of the detectors is in the fA range. Fig. 4 shows the capacitance of the $0.63 \mathrm{~mm}^{2}$ detector as plotted versus the reverse bias voltage. Subtracting a $0.5-\mathrm{pF}$ stray component, a capacitance of about $2 \mathrm{pF}$ in full depletion conditions is derived, in good agreement with the theoretical value dictated by the geometry and the electric permittivity of silicon carbide.

The front-end transistor used is a GaN high electron mobility transistor (HEMT) with a $100-\mu \mathrm{m}$-wide channel, realized by

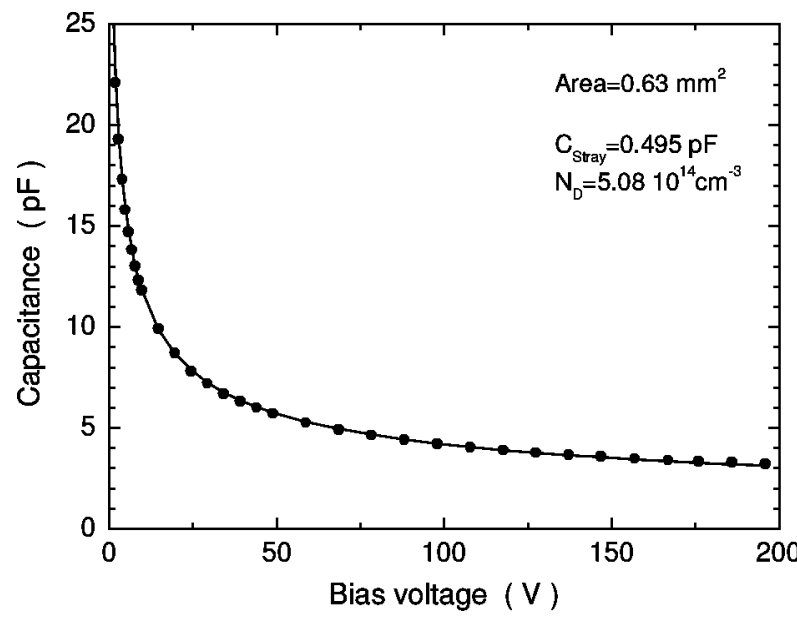

Fig. 4. Capacitance of the $0.63 \mathrm{~mm}^{2} \mathrm{SiC}$ detector as plotted versus the reverse bias voltage. Subtracting a $0.5 \mathrm{pF}$ stray component a capacitance of about $2 \mathrm{pF}$ is deduced, in good agreement with the theoretical value.

Italian company SELEX Integrated Systems [7]. Fig. 5 shows the drain current of two identical such devices as plotted versus the drain-to-source voltage. The drain current reaches very high values, up to $50 \mathrm{~mA}$. The transconductance, $\mathrm{g}_{\mathrm{m}}$, is plotted versus the gate-source voltage in Fig. 6. A peak value of $\sim 13 \mathrm{mS}$ is reached for $\mathrm{V}_{\mathrm{DS}}=3 \mathrm{~V}$ and $\mathrm{V}_{\mathrm{GS}}=-1.5 \mathrm{~V}$. The two transistors have similar performance, but a big difference is observed in their gate current, as shown in Fig. 7. So we used the low-current device called "HEMT2" in the spectrometer. We chose the bias point $\mathrm{V}_{\mathrm{DS}}=2.5 \mathrm{~V}, \mathrm{~V}_{\mathrm{GS}}=-1.5 \mathrm{~V}$, for the HEMT, which yields a large transconductance and a relatively low gate current. Note, however, that unfortunately the HEMT gate current is orders of magnitudes larger than the leakage current of the detector, which makes the HEMT intrinsically much more noisy than the detector itself. We found that such a high current is not related to the bulk current of the reverse-biased gate Schottky junction, but to the surface current through the leaky insulator layers currently used to isolate the electrical connections. So a major improvement is foreseen in this regard through the use of a better insulating layer. In Fig. 8, a measurement of the HEMT series noise is shown, which reveals a dominant $1 / \mathrm{f}$ component in a wide frequency range.

Using the noise parameters derived from the detector/HEMT characterization, we calculated the expected performance of the spectrometer. This is shown in Fig. 9. Assuming the use of the large $1.6 \mathrm{~mm}^{2}$ detector and considering that its capacitance is $\sim 8 \mathrm{pF}$, including the stray components, an equivalent noise charge (ENC) of $\sim 1400$ electrons r.m.s. at a shaping time of $\sim 100 \mathrm{~ns}$ is expected, which is still low enough for high-resolution $\alpha$-particle spectroscopy. It is worth pointing out that the dominant contribution to the ENC in this case comes from the HEMT $1 / \mathrm{f}$ noise enhanced by the relatively high value of the detector capacitance [8].

\section{MEASUREMENTS}

The available alpha particle source was a mixture of ${ }^{237} \mathrm{~Np}$, ${ }^{241} \mathrm{Am}$, and ${ }^{244} \mathrm{Cm}$ deposited on a radiopure substrate as a thin spot $0.5 \mathrm{~cm}$ wide in diameter. For safety reasons, the nominal 


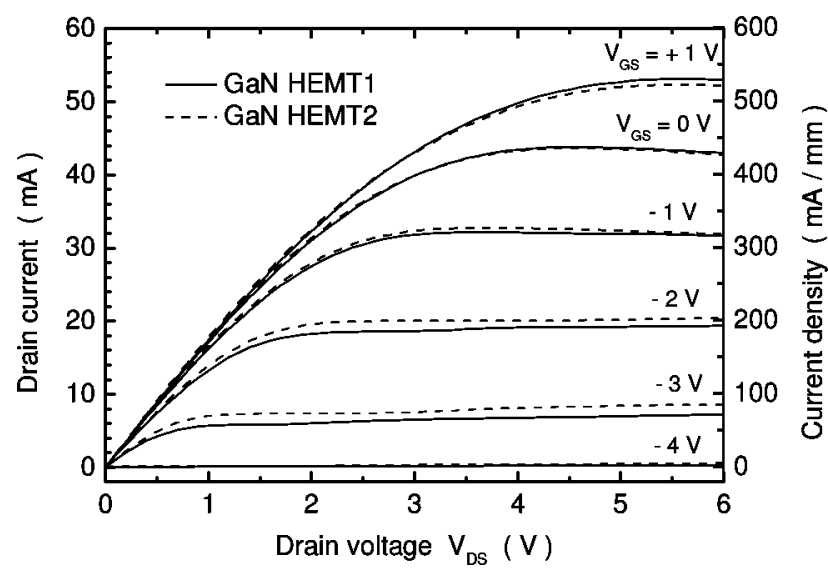

Fig. 5. Drain current of the used GaN HEMTs as plotted versus the drain-tosource voltage.

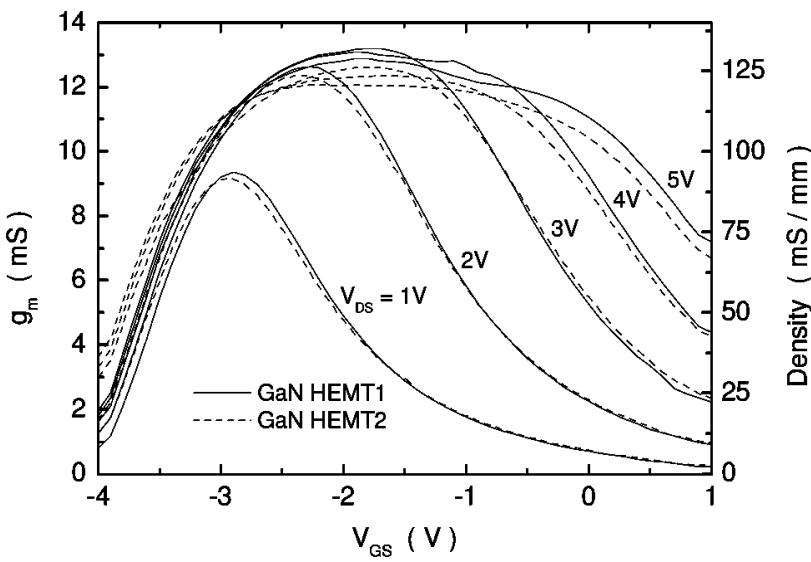

Fig. 6. Transconductance of the used GaN HEMTs as plotted versus the gate-to-source voltage.

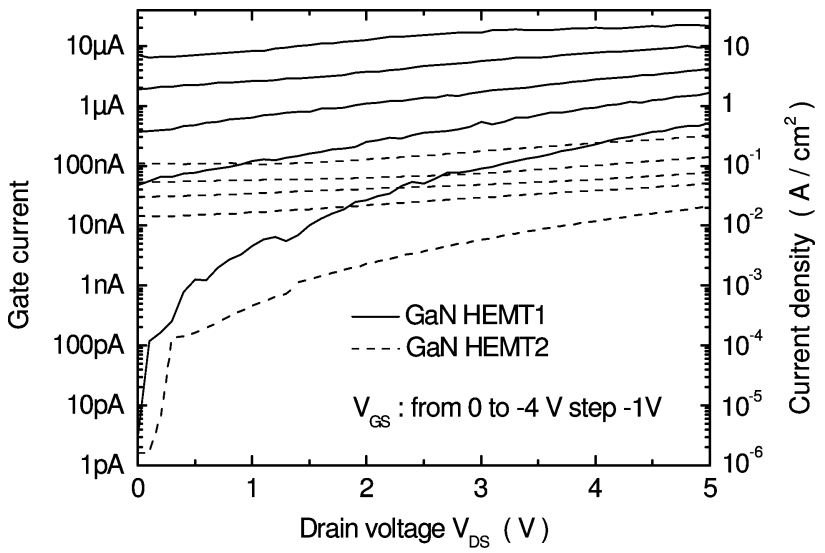

Fig. 7. Gate current of the GaN HEMTs.

activity of the source was only $100 \mathrm{~Bq}$. No encapsulation was present. Owing to the very low activity of the source we could use no collimator. In Table II the energies of the principal lines of the $\alpha$ source are shown. Due to the low activity of the source, we decided to employ the largest available detector (i.e., the $1.6 \mathrm{~mm}^{2}$ diode), obtaining a rate of detected events of $\sim 0.5$

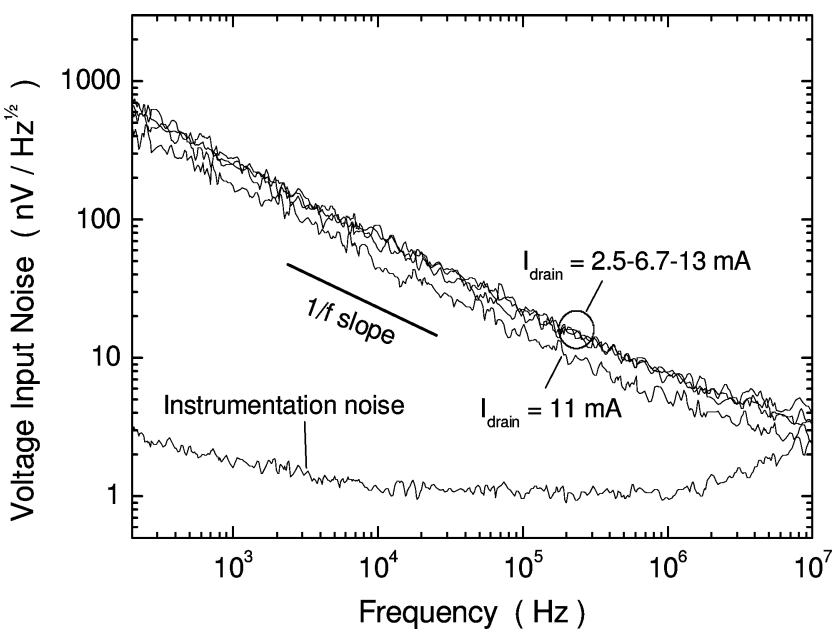

Fig. 8. Spectral density of series noise of HEMT2 as operated at different drain currents.

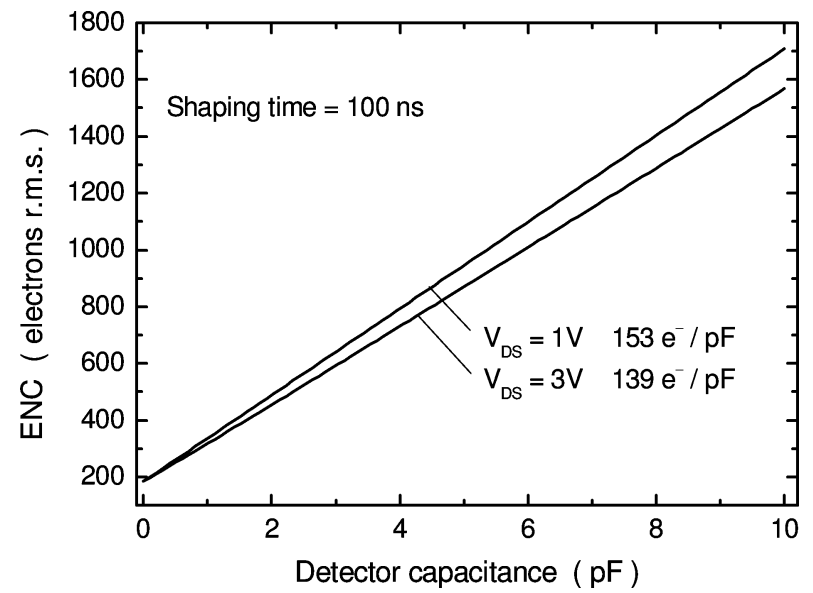

Fig. 9. Expected ENC versus detector capacitance of the GaN HEMT preamplifier.

TABLE II

PRINCIPAL LiNES OF THE $\alpha$ SOURCE

\begin{tabular}{l|c|c|c|c|c|c|c|c}
\hline Radionuclide & \multicolumn{4}{|c|}{${ }^{237} \mathrm{~Np}$} & \multicolumn{2}{|c|}{${ }^{241} \mathrm{Am}$} & \multicolumn{2}{|c}{${ }^{244} \mathrm{Cm}$} \\
\hline Energy (MeV) & 4.639 & 4.771 & 4.788 & 4.872 & 5.443 & 5.486 & 5.763 & 5.805 \\
\hline Probability (\%) & 6 & 25 & 47 & 1.4 & 12.8 & 85.2 & 23.6 & 76.4 \\
\hline
\end{tabular}

events/s when placing the source at a distance of $\sim 5 \mathrm{~mm}$ from the detector.

As a preliminary measurement, we collected a sequence of spectra of the source at increasing values of the detector bias voltage to check if the detector was able to stop all the emitted $\alpha$ particles. To do this, we used a simple front-end circuit using a junction field effect transistor (JFET) as its input transistor. The obtained results are shown in Fig. 10. It can be seen that the principal lines of the $\alpha$ source show up one by one when increasing the bias voltage from 60 to $180 \mathrm{~V}$. This depends on the increasing depth of the detector depletion layer which sequentially reaches the Bragg-peak position of the $\alpha$ particles traveling through the detector. As shown in Fig. 11, it can also be noted that the position of the peaks is not appreciably shifting when increasing the bias voltage from 180 to $300 \mathrm{~V}$, which 


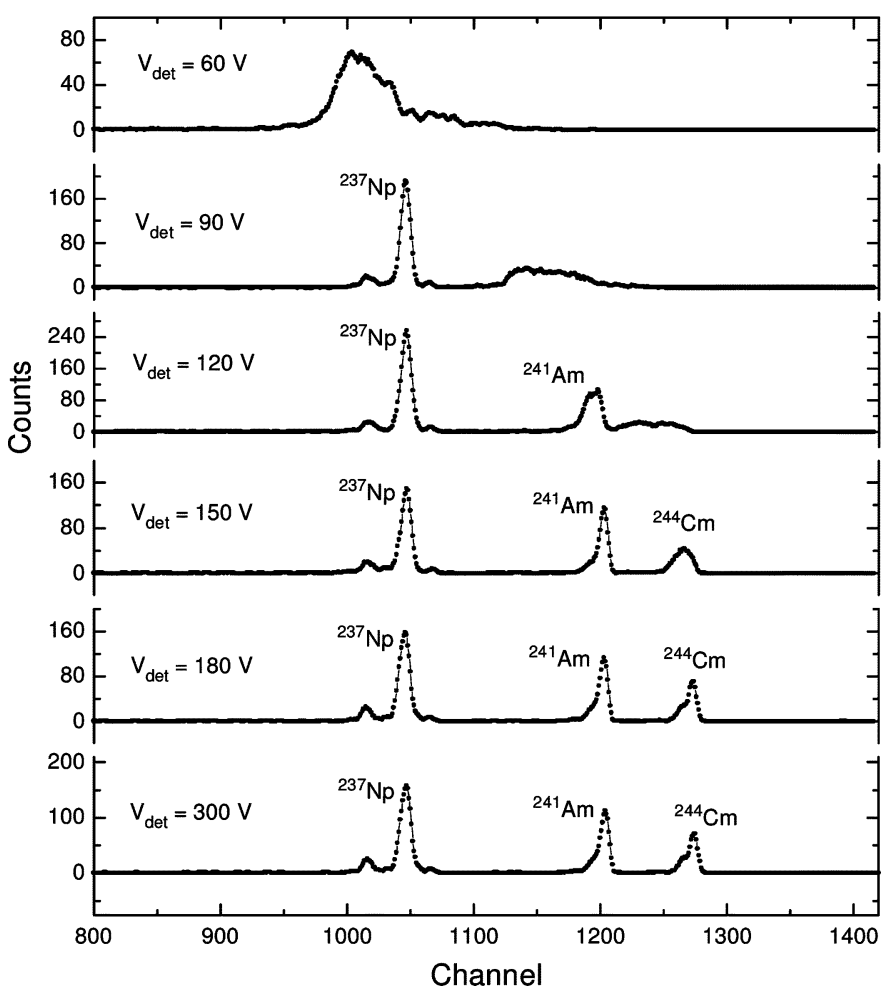

Fig. 10. Spectra collected at increasing values of the detector bias voltage.

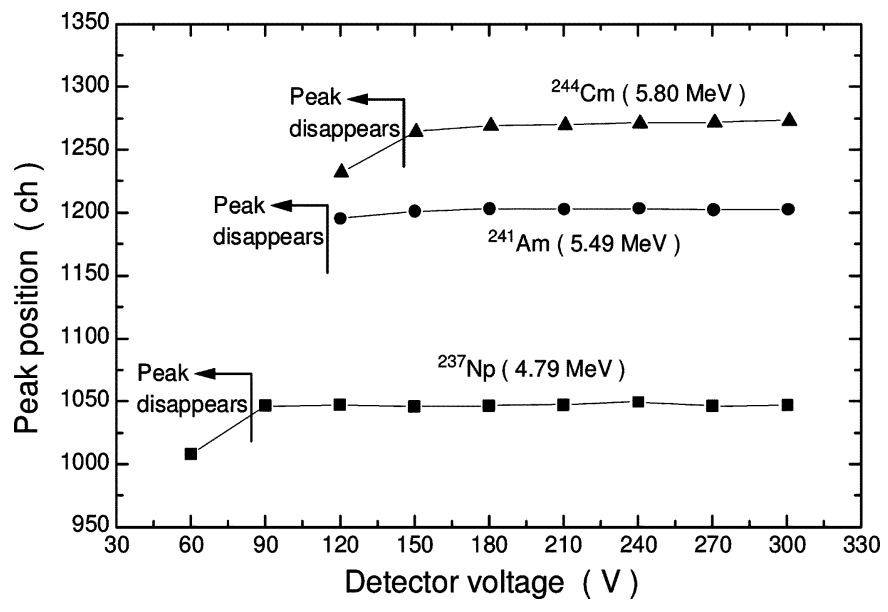

Fig. 11. Position of the spectral lines at increasing detector bias voltage.

means that the collection efficiency is full as soon as the Bragg peak entirely falls into the depleted region of the detector.

\section{AsSEMbly OF THE SyStem AND EXPERIMENTAL RESUltS}

We eventually designed and realized a custom charge-sensitive preamplifier that is able to exploit the peculiar characteristics of the GaN HEMT shown in Section II. In such a preamplifier, the bias point of the HEMT was forced to $\mathrm{V}_{\mathrm{DS}}=2.5 \mathrm{~V}, \mathrm{~V}_{\mathrm{GS}}=-1.5 \mathrm{~V}$, so as to maximize its transconductance. The system was housed in a two-cavity metallic box. In the first cavity, we placed the principal devices (i.e., the $\mathrm{SiC}$ detector), the GaN HEMT and the $\alpha$-particle source. In the second cavity, we placed the remaining part of the preamplifier.

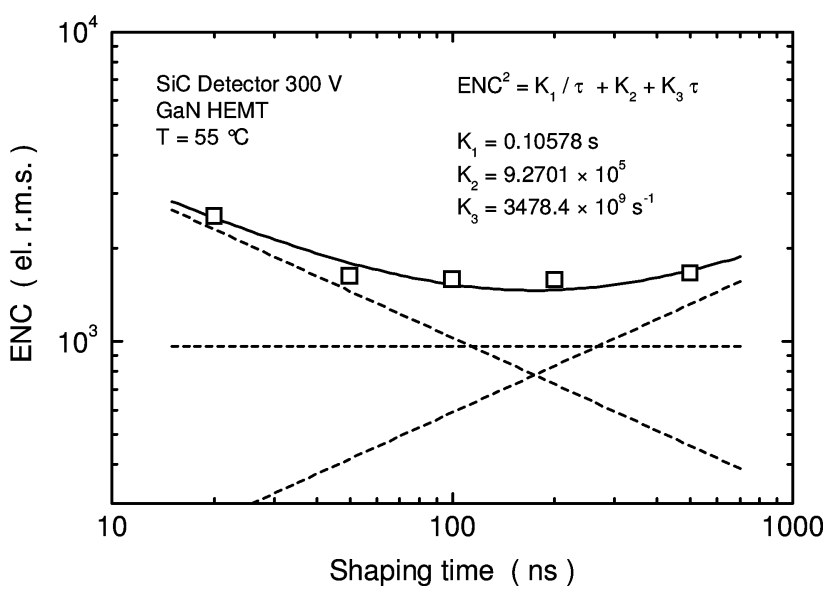

Fig. 12. ENC versus shaping time and fitting curve in solid line. The dashed lines are the disentangled noise contributions.

The electrical interconnection between the two parts was made through twisted pair cables passing through a fissure in the floor of the first cavity. Such a setup was adopted in order to minimize the electromagnetic interference and noise picked up by the detector/HEMT system. Special care was devoted to providing suitable return-current ground paths to the electrical connections between the HEMT and the remaining part of the preamplifier, which greatly helped stabilize the system and reduce the electromagnetic interference (EMI) [9]. The entire box was then fitted in a vacuum chamber so as to eliminate any absorption or energy loss of the $\alpha$ particles traveling through the air.

With the $1.6 \mathrm{~mm}^{2}$ detector connected, we obtained a preamplifier risetime of $\sim 8 \mathrm{~ns}$ and an ENC of $\sim 1500 \mathrm{rms}$ electrons at a temperature of $+55^{\circ} \mathrm{C}$ and a RC-CR peaking time of $100 \mathrm{~ns}$. The graph of ENC versus shaping time is shown in Fig. 12. Considering that the detector capacitance, including the stray components, is of $\sim 8 \mathrm{pF}$, the obtained ENC is in sufficient agreement with the calculated value predicted by the graph of Fig. 8 . It is worth noting that the ENC does not change that much in the shaping time range of 100 to $500 \mathrm{~ns}$.

We finally acquired some spectra using a Gaussian shaper Ortec 572 as set at a shaping time of $500 \mathrm{~ns}$. We could not measure data at shorter shaping times because the specifications of the available peak ADC did not cover any shorter shaping time range. In Fig. 13, the best acquired spectrum is shown, along with a fitting of the principal lines and the pulser line. The energy resolution observed on the pulser line at an operation temperature of $55{ }^{\circ} \mathrm{C}$ is of $29 \mathrm{keV}$ f.w.h.m. or 1500 electrons rms, in agreement with the data of Fig. 12. The energy resolution on the principal $\alpha$ particles lines is shown in Table III and is below $1 \%$.

We found that similar to [1] in our case, the main contribution to the line width is neither the electronic noise nor the intrinsic contribution due to the Fano factor, but the statistics of the energy loss in the gold contact acting as entrance windows for the $\alpha$ particles. The use of no collimator made the dispersion of such energy loss wider, as the $\alpha$ particles could enter the detector along angled trajectories, thus having to traverse different widths of gold before reaching the semiconductor. This 


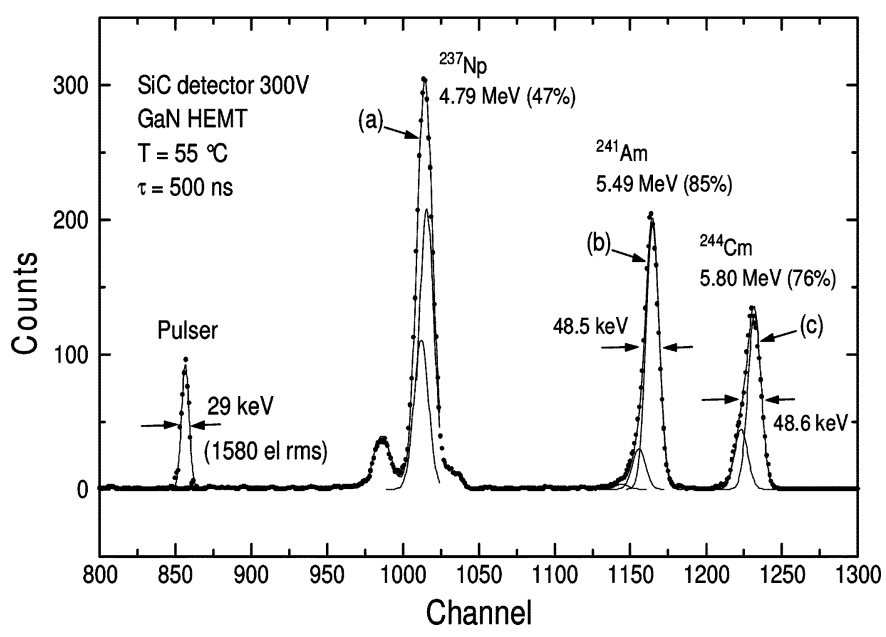

Fig. 13. Spectrum of the alpha source obtained with the $\mathrm{SiC} / \mathrm{GaN}$ system. The substructure of the spectral lines is shown as obtained through a Gaussian fitting procedure.

TABLE III

SPECTRUM ANALYSIS

\begin{tabular}{cccc}
\hline Spectral line & Energy & $\begin{array}{c}\text { Line width } \\
\text { FWHM }\end{array}$ & Resolution \\
\hline (a) & $4.79 \mathrm{MeV}$ & $48 \mathrm{keV}$ & $1 \%$ \\
\hline (b) & $5.49 \mathrm{MeV}$ & $48.5 \mathrm{keV}$ & $0.88 \%$ \\
\hline (c) & $5.80 \mathrm{MeV}$ & $48.6 \mathrm{keV}$ & $0.84 \%$ \\
\hline Pulser & - & $29 \mathrm{keV}$ & - \\
\hline
\end{tabular}

effect became more evident when placing the source at a closer distance of only $0.5 \mathrm{~mm}$ to the detector. In this case, the energy resolution worsened remarkably as the angular distribution of the possible trajectories was much broader. Unfortunately, we could not try the opposite (i.e., place the source at a large distance from the detector and/or use a collimator), because of the very low activity of the source. We believe, however, that a reduced thickness for the Au contact as well as the use of a collimator would help improve the resolution. A significant reduction of the electronic noise could moreover be achieved using next-generation GaN HEMTs, with lower gate leakage current and reduced $1 / \mathrm{f}$ noise.

\section{CONCLUSION}

We designed, realized, and tested an $\alpha$-particle spectrometer based on a $4 \mathrm{H} \mathrm{SiC} \mathrm{detector} \mathrm{and} \mathrm{a} \mathrm{GaN} \mathrm{HEMT} \mathrm{front-end} \mathrm{tran-}$ sistor. We obtained a high resolution of the order of $0.9 \%$ in the 5
$\mathrm{MeV}$ energy range at $55^{\circ} \mathrm{C}$, which is a significant new achievement using wide bandgap semiconductors for the detector/frontend-electronics system. We believe that such a system can have unique applications. For example, nuclear reactor power monitoring must be carried out in a high-temperature environments. Although not yet demonstrated (to our knowledge), $\mathrm{GaN}$ is also likely to have the same radiation resistance properties that have been demonstrated for $\mathrm{SiC}$. Therefore, the $\mathrm{SiC} / \mathrm{GaN}$ device may be useful for prolonged measurements in high radiation environments as well as at high temperatures. Currently, the limiting factor of the system performance is the energy loss in the gold Schottky contact acting as entrance window for the $\alpha$ particles. Use of a thinner Schottky barrier made, for example, by nickel silicide would likely yield even better energy resolution. The dominant contributions to the ENC are the 1/f noise of the HEMT and the shot noise of the HEMT's gate current.

\section{ACKNOWLEDGMENT}

The authors would like to thank C. Lanzieri, F. Nava, S. Sivero, S. Masci, R. Peroni, and S. Lavanga.

\section{REFERENCES}

[1] F. H. Ruddy, J. G. Seidel, H. Chen, A. R. Dulloo, and S. Hyung Ryu, "High-resolution alpha-particle spectrometry using $4 \mathrm{H}$ silicon carbide semiconductor detectors," IEEE Trans. Nucl. Sci., vol. 53, no. 3, pt. 3, pp. 1713-1718, Jun. 2006.

[2] F. Nava, P. Vanni, M. Bruzzi, S. Lagomarsino, S. Sciortino, G. Wagner, and C. Lanzieri, "Minimum ionizing and alpha particels detectors based on epitaxial semiconductor silicon carbide," IEEE Trans. Nucl. Sci., vol. 51, no. 1, pt. 2, pp. 238-244, Feb. 2004.

[3] G. Bertuccio and R. Casiraghi, "Study of silicon carbide for X-ray detection and spectroscopy," IEEE Trans. Nucl. Sci., vol. 50, no. 1, pt. 2, pp. 175-185, Feb. 2003.

[4] B. F. Phlips, K. D. Hobart, F. J. Kub, R. E. Stahlbush, M. K. Das, B. A. Hull, G. De Geronimo, and P. O'Connor, "Silicon carbide PiN diodes as radiation detectors," in Proc. IEEE Nucl. Sci. Symp. Conf. Rec., Oct. 23-29, 2005, vol. 3, pp. 1236-1239.

[5] G. Bertuccio, S. Caccia, R. Casiraghi, and C. Lanzieri, "Possibility of sub-electron noise with room temperature silicon carbide pixel detectors," IEEE Trans. Nucl. Sci., vol. 53, no. 4, pp. 2421-2427, Aug. 2006.

[6] G. Bertuccio, S. Caccia, A. Pullia, D. Maiocchi, F. Nava, C. Lanzieri, and S. Lavanga, "Design of a SiC/GaN heterodevice for ionizing radiation detection," presented at the 31st Workshop on Compound Semiconductor Devices and Integrated Circuits, Venice, Italy, May 20-23, 2007, WOCSDICE 2007.

[7] SELEX Sistemi Integrati S.p.A. Via Tiburtina km 12 400, 00131, Roma.

[8] E. Gatti, P. F. Manfredi, M. Sampietro, and V. Speziali, "Suboptimal filtering of $1 / \mathrm{f}$ noise in detector charge measurements," Nucl. Instr. Meth., vol. A297, pp. 467-478, 1990.

[9] H. W. Ott, Noise Reduction Techniques in Electronic Systems. New York: Wiley, 1976, p. 140.

[10] "Development of a SiC/GaN detector/front-end integrated system for ionizing radiation spectroscopy at room and high temperatures." 2005. [Online]. Available: http://www.ricercaitaliana.it/prin/dettaglio_completo_prin_en-2004027857.htm 